\title{
IMPACTO DE UMA DÉCADA DE UM PROGRAMA DE BASE COMUNITÁRIA EM MEIO ESCOLAR, NA OBESIDADE INFANTIL EM PORTUGAL: MUN-SI 2008-2018
}

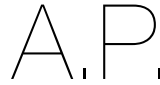

ARTIGOPROFSSIONAL

${ }^{1}$ Instituto Nacional de Saúde Doutor Ricardo Jorge,

Avenida Padre Cruz, 1649-016 Lisboa, Portugal

${ }^{2}$ Centro de Estudos e Investigação em Dinâmicas Sociais e Saúde, Avenida Padre Cruz, 1649-016 Lisboa, Portugal

"Endereço para correspondência: Délfia Lopes Centro de Estudos Contro do Estudos e nâmicas 49-016 Lisboa, Portugal delfialopess@gmail.com

Histórico do artigo:

\section{IMPACT OF A COMMUNITY-BASED PROGRAM DURING A DECADE IN SCHOOLS SETTING ON CHILDHOOD OBESITY} IN PORTUGAL: MUN-SI 2008-2018
RESUMO

INTRODUÇÃo: Em Portugal, a Obesidade destaca-se como a doença mais prevalente na infância, com 1 em cada 3 crianças com excesso de peso, ainda que pareça observar-se uma tendência invertida na última década. Programas contínuos, de base comunitária ao nível local parecem responder de forma eficaz a esta problemática. O MUN-SI surgiu em 2008, como um programa de promoção da saúde infantil em municípios com o objetivo de retardar o excesso de peso infantil de forma interativa.

OBJETIVOS: Avaliar o impacto do MUN-SI no estado nutricional, a mudança comportamental e de hábitos alimentares, a aquisição de conhecimentos, a adesão à Dieta Mediterrânica e a prática de atividade física, em crianças dos 6 aos 10 anos, entre 2008 e 2018 , no Município de Oeiras.

METODOLOGIA: O MUN-Si intervém através de uma matriz de 3 parâmetros: promover hábitos alimentares saudáveis de acordo com a temática a abordar, promover a prática de atividade física regular e avaliar o estado nutricional infantil, em escolas do $1 .{ }^{\circ}$ Ciclo do Ensino Básico. As sessões de intervenção lúdico-educativas em sala de aula foram implementadas por nutricionistas treinados e incluíram: diagnóstico inicial e sensibilização para a temática, intervenção motivacional e avaliação do programa com participação ativa da família. Os comportamentos alimentares e prática de atividade física foram avaliados através de um questionário à família. $\mathrm{O}$ estado nutricional infantil foi classificado pelos critérios da Organização Mundial da Saúde, 2007.

RESULTADOS: Registou-se uma mudança comportamental positiva na preferência de inclusão de hortofrutícolas nos lanches (+2,3\% em 2015/2016) e ao pequeno-almoço (+16\% em 2014/2015 e +11,9\% em 2017/2018). A aquisição de conhecimentos sobre a Dieta Mediterrânica foi verificada em +12,1\% a 21,1\%, entre 2016 e 2019 e a adesão à Dieta Mediterrânica aumentou em 20,9\%, em 2016/2017. Houve um aumento na prática de atividade física regular (11 a 15\%) e uma redução de 11,2\% na prevalência excesso de peso (35,5\% para $24,3 \%$ ) e $10,3 \%$ de obesidade $(16,7 \%$ para $6,4 \%)(p<0,005)$.

CONCLUSÕES: Os resultados do MUN-SI sugerem que programas de base comunitária multidisciplinares integrados e aplicados continuamente ao nível local, têm efeito na adoção de estilos de vida mais saudáveis e na diminuição da prevalência de excesso de peso e obesidade infantil.

\section{PALAVRAS-CHAVE}

Municípios, Obesidade infantil, Programa de base escolar, Programa de nutrição e saúde comunitária

\section{ABSTRACT}

INTRODUCTION: In Portugal, obesity is the most prevalent disease in childhood, with 1 in every 3 children being overweight, although in the last decade it has been showing a downward trend. Ongoing, community-based programs at local level seem to respond effectively to this problem. MUN-SI emerged in 2008 as a program to promote child health in municipalities with the aim of interactively tackle childhood overweight and obesity.

OBJECTIVES: Impact assessment of MUN-SI program on the nutritional status, on attitudes and behaviors regarding diet, on knowledge and adherence to the Mediterranean Diet and on physical activity, of children aged 6 to 10 years, between 2008 and 2018 , in the municipality of Oeiras.

METHODOLOGY: MUN-SI intervenes through a matrix of 3 parameters: to promote healthy eating habits according to the selected theme, to promote regular physical activity, and to assess the nutritional status of children in primary schools, after the intervention. The playfuleducational intervention classroom sessions were implemented by trained nutritionists and included: initial diagnosis and awareness of the theme, motivational intervention and evaluation of the program with active family participation. Eating behaviors and physical activity were assessed using a family questionnaire. Child nutritional status was classified according to World Health Organization, 2007 criteria. RESULTS: There was a positive behavioral change in the preference for including fruit and vegetables in home brought to school lunches (+ 2.3\% in 2015/2016) and at breakfast (+ 16\% in 2014/2015 and +11.9\% in 2017/2018). The acquisition of knowledge about the Mediterranean Diet was observed in $+12.1 \%$ to $21.1 \%$ of the children, between 2016 and 2019 and the adherence to Mediterranean Diet increased in $20,9 \%$ of the children in $2006 / 2017$. There was an increase in regular physical activity (11 to $15 \%$ ) and a reduction of 11 , $2 \%$ in the prevalence of overweight (35.5\% to $24.3 \%$ ) and $10.3 \%$ of obesity $(16.7 \%$ to $6.4 \%)(p<0.005)$, during the decade of the study. CONCLUSIONS: The results of MUN-SI program suggest that integrated, multidisciplinary community-based programs on a continuous basis, at local level, have an effect on the adoption of healthier lifestyles by school age children and on decreasing the prevalence of overweight and childhood obesity. 
KEYWORDS

Municipalities, Childhood obesity, School-based program, Nutrition and health community program

\section{INTRODUÇÃo}

Em Portugal, os últimos dez anos foram pautados pelo reconhecimento que a obesidade Infantil continua a ser a doença mais prevalente na infância e um importante problema de saúde pública (1). O estudo Childhood Obesity Surveillance Initiative (COSI) da Organização Mundial da Saúde (OMS)/Europa (2), que Portugal lidera desde 2008, tem vindo a mostrar consistentemente uma tendência invertida da prevalência de excesso de peso e obesidade infantil. De 2008 (1. ${ }^{a}$ edição do COSI Portugal) para 2019, verificou-se uma redução de 8,3\% na prevalência de excesso peso (37,9\% para 29,6\%) e de obesidade infantil de $15,3 \%$ (2008) para 12,0\% em 2019 (3,4). Contudo, apesar deste indicador positivo, Portugal ainda se encontra no grupo de países Europeus com maior prevalência de excesso de peso infantil e a maioria destas crianças cresce em ambientes obesogénicos (5-7).

A revogabilidade desta situação e a sua expressão, justificam a prioridade atribuível à formulação e ao desenvolvimento de programas de ação que estimulem a adoção de estilos de vida mais saudáveis e criem condições estruturais e ambientais favoráveis à saúde. Neste contexto, programas de base comunitária, realizados ao nível local e integrados nas políticas de saúde regionais, guiados por programas e políticas nacionais e internacionais, parecem responder de forma eficaz a esta problemática (8). A ação deve ser realizada tanto ao nível micro como macro e em diferentes contextos (familiar, comunitário e escolar) (9).

Intervir na infância traduz uma oportunidade para efeitos na saúde mais sustentáveis resultantes de uma maior predisposição para a mudança e aquisição de estilos de vida saudáveis (10). Esta condição é ainda mais pertinente no grupo etário dos 6-10 anos, já que é uma fase pré-pubertária e uma idade bastante recetiva e interventiva no seio familiar constituindo um momento crucial para consolidar hábitos alimentares e estilos de vida saudáveis (2).

A revisão Bemelmans et al. (11) de 71 programas de base comunitária reforça ainda que intervenções bem sucedidas são aquelas que combinam continuidade com multidisciplinaridade, envolvendo os participantes e agentes nas etapas de planeamento e implementação, transversais aos vários setores da comunidade, compreensivas, adaptadas ao contexto local e usando as estruturas locais existentes $(11,12)$ como são as escolas, enquanto locais privilegiados de promoção da saúde infantil (10).

Neste enquadramento, o MUN-SI (13) surgiu em 2008 como um programa contínuo de intervenção comunitária, de base municipal, com o objetivo de retardar a progressão da prevalência da pré-obesidade e da obesidade infantil em Portugal, oferecendo uma resposta interventiva e interativa a esta problemática. Um programa desenvolvido através de uma abordagem compreensiva e multidisciplinar que pretende construir um modelo ecológico comunitário e sustentável. Estabelecendo prioridades de saúde locais com os municípios, e com base em ações contínuas, é dirigido a crianças em idade escolar, através de atividades específicas de promoção de hábitos saudáveis de vida em participação ativa do ambiente escolar. Pretende ainda ser decisivo nas decisões políticas locais para a promoção e a incorporação da saúde nas atividades de desenvolvimento comunitário, em particular no que diz respeito aos hábitos alimentares e à promoção de atividade física na infância e em todos os seus planos mais adjacentes (família e escola).

O programa MUN-SI tem vindo a ser implementado consecutivamente nos últimos 10 anos em vários municípios: nos anos 2008 a 2011 em Viana do Castelo, Seixal, Fundão e Montijo, nos anos 2017 a 2019 em Cascais e desde 2008, continuamente em Oeiras. O programa envolveu, até 2018, um total de 186 escolas do $1 .{ }^{\circ}$ Ciclo do Ensino Básico, abrangendo 390 professores e 9754 crianças, com idades compreendidas entre os 6 e 12 anos.

O objetivo do presente artigo é avaliar o impacto do Programa MUN-SI, nos comportamentos e práticas alimentares, ao nível da prática de atividade física e do estado nutricional de crianças dos 6 aos 10 anos, entre 2008 e 2018, no Município de Oeiras.

\section{METODOLOGIA}

O programa MUN-SI é dirigido a toda a comunidade educativa do $1 .{ }^{\circ}$ Ciclo do Ensino Básico do Município participante e desenvolve-se com uma matriz comum de 3 parâmetros: promoção de hábitos alimentares saudáveis, promoção de atividade física e avaliação do estado nutricional em crianças dos 6 aos 10 anos. O programa é desenhado anualmente de acordo com necessidades locais identificadas pelo município. A estratégia de implementação do programa para cada ano letivo inicia-se com o desenvolvimento de um protocolo metodológico acordado pela equipa coordenadora MUN-SI, que para além dos Nutricionistas Supervisores, inclui os técnicos municipais indicados para o Programa e, após seleção das Escolas, os professores dos anos de escolaridade abrangidos.

\section{População em Estudo}

A seleção de Escolas do 1. ${ }^{\circ}$ Ciclo do Ensino Básico (1CEB) foi realizada, em cada ano letivo, através de carta de apresentação do programa conjunta com município, de convite às 37 escolas das 10 freguesias de Oeiras. O número de escolas participantes, anos de escolaridade abrangidos e número total de alunos inscritos no Programa, está apresentado na Tabela 1.

\section{A Intervenção}

Em cada ano letivo, decorreram três momentos de intervenção implementadas por 4-5 profissionais que foram treinados nos procedimentos metodológicos padrão do programa MUN-SI. Nos 10 anos do programa, foram treinados 60 profissionais dos quais 52 eram nutricionistas e os restantes estudantes, professores e técnicos municipais.

\section{Tabela 1}

$\mathrm{N} .^{\circ}$ de Escolas do $1 .^{\circ}$ ciclo do ensino básico de Oeiras participantes no programa MUN-SI, anos de escolaridade abrangidos e total de crianças inscritas por ano de programa de 2008 a 2019

\begin{tabular}{|c|c|c|c|c|c|c|c|c|c|}
\hline & $2008 / 2009$ & $2010 / 2011$ & $2012 / 2013$ & $2013 / 2014$ & $2014 / 2015$ & $2015 / 2016$ & $2016 / 2017$ & $2017 / 2018$ & $2018 / 2019$ \\
\hline $\begin{array}{l}\text { Total de escolas do } 1 .^{\circ} \text { ciclo do ensino } \\
\text { básico de Oeiras }\end{array}$ & 30 & 27 & 8 & 6 & 7 & 8 & 7 & 5 & 3 \\
\hline $\begin{array}{l}\text { Anos de escolaridade abrangidos pelo } \\
\text { programa }\end{array}$ & $1^{\circ}, 2^{\circ}$ & $3^{\circ}, 4^{\circ}$ & $1^{\circ}, 2^{\circ}, 3^{\circ}, 4^{\circ}$ & $1^{\circ}, 2^{\circ}, 3^{\circ}, 4^{\circ}$ & $1^{\circ}, 2^{\circ}, 3^{\circ}, 4^{\circ}$ & $1^{\circ}, 2^{\circ}, 3^{\circ}, 4^{\circ}$ & $3^{\circ}$ & $4^{\circ}$ & $3^{\circ}, 4^{\circ}$ \\
\hline Total de crianças inscritas no programa & 1167 & 1057 & 480 & 640 & 1630 & 1750 & 371 & 281 & 320 \\
\hline
\end{tabular}


O primeiro momento de intervenção teve o carácter de diagnóstico inicial e momento lúdico-educativo para sensibilização da temática a abordar, o segundo momento foi de intervenção motivacional e o terceiro momento de avaliação do programa, com participação ativa da família. Os pressupostos da intervenção centraram-se na definição do problema, em técnicas de motivação e sensibilização para a mudança de hábitos e comportamentos, no foco nas competências existentes (empowerment) e ainda em estratégias de resolução das situações apresentadas.

Cada sessão teve a duração de cerca de 60 minutos e realizou-se habitualmente em sala de aula ou em espaço escolar indicado (refeitório ou recreio). As sessões foram dirigidas a cada turma de alunos nas escolas participante sendo que na primeira e na terceira sessão ocorreu preenchimento dos instrumentos de avaliação,

O foco da intervenção foi a promoção da alimentação saudável e a prática de atividade física. Muito embora o objetivo fosse abordar as questões relacionadas com a obesidade infantil e a escola e família tivessem conhecimento destes objetivos, esta problemática nunca foi destacada nem as sessões se centraram à volta do estado nutricional infantil.

Independentemente da temática alimentar selecionada, os conteúdos foram transmitidos valorizando o conceito e os princípios da Dieta Mediterrânica, promovendo um estilo de vida saudável e equilibrado, com um padrão alimentar caracterizado por uma alimentação simples, completa, variada e equilibrada, com preferência por alimentos locais e sazonais, tendo em consideração a sustentabilidade e o meio ambiente e ao mesmo tempo promotora dos aspetos culturais que e celebra o convívio em volta da mesa.

Foram igualmente destacadas as oportunidades de realizar e aumentar a prática de exercício físico, para além daquelas atualmente oferecidas em cada escola. A intervenção focou-se em reforçar a atividade física espontânea nos espaços escolares como no recreio, à hora do almoço e depois da escola, integrada num estilo de vida tendencialmente mais ativo como jogos ao ar livre, ciclismo, caminhadas na praia e jogos infantis.

A participação da família foi crucial durante toda fase interventiva concentrando-se esta no estímulo e apoio dos pais como elementos chave para a mudança comportamental. O programa promoveu sessões de educação de teor prático, em conjunto com as crianças, em ambiente escolar.

A abordagem do Programa MUN-SI integrou continuamente os componentes lúdico-educativos (jogos, dinâmicas de grupo, workshops, gincanas, quizzes, showcookings) que foram construídos com todos os agentes participativos: crianças, professores pais e comunidade escolar.

\section{Mudança de comportamentos, conhecimentos e atitudes alimentares}

Um questionário validado (14) para crianças portuguesas que incluía perguntas sobre conhecimentos, atitudes e comportamentos nutricionais, foi preenchido pelas crianças com a ajuda dos nutricionistas. O instrumento era adaptado do original compreendendo apenas as perguntas relativas às temáticas de cada ano do programa. Um sistema de pontuação deu a cada resposta correta (melhoria nos conhecimentos adquiridos e atitude positiva, ou mudança comportamental) um ponto, tendo sido atribuído zero sempre que não se registou melhoria ou a alteração de hábitos.

\section{Atividade física}

A atividade física foi avaliada através de um questionário dirigido à família (14), sobre a frequência semanal de exercício físico/desporto organizado. Os dados reportados foram agrupados em 3 categorias de 0-2 vezes/semana de 3-6 vezes/semana e todos os dias.

\section{Estado Nutricional}

Para avaliação e classificação do estado nutricional infantil foram consideradas as medidas antropométricas de peso e estatura. A avaliação antropométrica foi realizada após o consentimento informado do encarregado de educação e da criança e seguiu as orientações específicas que constavam no protocolo de intervenção MUN-SI que compreendem as diretrizes do estudo COSI da OMS/Europa $(2,15)$. O estado nutricional das crianças foi definido de acordo com os critério de referência da OMS 2007 (16), que define Baixo peso/idade, pré-obesidade e obesidade como um Índice de Massa Corporal (IMC) para idade de $<-2$ desvio-padrão (DP), $+1<\mathrm{DP}<+2$ (equivalente a um IMC de $25 \mathrm{~kg} \mathrm{~m}^{2}$ aos 19 anos) e > +2 DP (equivalente a um IMC de $30 \mathrm{~kg} \mathrm{~m}^{2}$ aos 19 anos), respetivamente.

As considerações éticas do programa MUN-SI foram apresentadas e aprovadas anualmente pelo Município de Oeiras, bem como pela Direção de cada escola interveniente.

\section{RESULTADOS}

Os temas abordados e o impacto do programa MUN-SI na mudança comportamental, nos conhecimentos adquiridos e adesão à Dieta Mediterrânica (DM) atitudes e na prática de atividade física das crianças participantes, em cada ano letivo, estão apresentados na Tabela 2.

Nos anos letivos 2013/2014 a 2015/2016, foi abordada a temática dos lanches saudáveis. Nestes primeiros 2 anos escolares não se verificam efeitos na mudança comportamental das crianças. Em 2015/2016, após intervenção, observou-se uma melhoria nas escolhas dos alimentos dos lanches trazidos para a escola, tendo-se verificado uma diminuição de alimentos açucarados (pão de leite, bolos e bolachas, sumos e refrigerantes: -6,0\%) e um aumento de hortofrutícolas (+2,3\%). Em relação à temática do "pequeno-almoço", esta foi abordada nos anos 2014/2015 e $2018 / 2019$. O aumento da inclusão de fruta ao pequeno-almoço foi um dos parâmetros mais reportado naqueles anos (+16\% em 2014/2015 e +11,9\% em 2017/2018). A Dieta Mediterrânica foi o tema central dos últimos 3 anos escolares (de 2016 a 2019) e permitiu avaliar tanto os parâmetros de mudança comportamental como ganhos na aquisição de conhecimentos. A adesão à DM, avaliado através do Kidmed (17), aumentou após as intervenções em 20,9\%, em 2016/2017. Da mesma forma houve um aumento nos conhecimentos sobre a DM: $21,1 \%$ no ano 2016/2017; $12,1 \%$ no ano 2017/2018 e 20,2\% em 2018/2019.

Em relação à promoção da prática de atividade física (AF), de acordo com as recomendações (18), entendeu-se como prática de AF regular uma frequência superior a 3 vezes por semana. Verificou-se que no ano 2017/2018 houve um aumento superior a 15\% de crianças que reportaram praticar AF regular, após 12 meses de intervenção, e nos anos 2010/2011 e 2018/2019 este efeito positivo verificou-se em $11 \%$ a $15 \%$ das crianças, respetivamente.

As características antropométricas (estatura, peso e IMC) estão representadas na Tabela 3.

Relativamente à estatura verificou-se que entre sexos os valores médios são semelhantes. Observou-se um aumento na estatura média quer nos rapazes (de 129,0 cm para 135,5 cm) quer nas raparigas (128,0 cm para 134,2 cm) e igualmente no peso médio: rapazes: de $29,0 \mathrm{~kg}$ para $30,9 \mathrm{~kg}$ e raparigas de 29,3 kg para $31,2 \mathrm{~kg}$, na década em estudo (2008/2019 a 2008/2019).

Constatou-se ainda que em todos os anos o sexo feminino apresentou 
Descrição dos temas e resultados da intervenção (6-8 meses ou 12 meses), do programa MUN-SI, na mudança comportamental, de conhecimentos e atitudes alimentares e na prática de atividade física, de 2008-2019

\begin{tabular}{|c|c|c|c|c|c|c|c|c|c|}
\hline \multirow[b]{2}{*}{ ANO LETIVO } & \multirow[b]{2}{*}{ TEMA } & \multicolumn{4}{|c|}{ PARÂMETROS ALIMENTARES } & \multicolumn{4}{|c|}{ ATIVIDADE FÍSICA } \\
\hline & & MUDANÇA COMPORTAMENTAL & EFEITO & $\begin{array}{l}\text { CONHECIMENTOS } \\
\text { E ATITUDES }\end{array}$ & EFEITO & $\begin{array}{l}\text { 0-2 VEZES/ } \\
\text { SEMANA }\end{array}$ & $\begin{array}{l}\text { 3-6 VEZES/ } \\
\text { SEMANA }\end{array}$ & $\begin{array}{l}\text { TODOS OS } \\
\text { DIAS }\end{array}$ & EFEITO \\
\hline $2008 / 2009$ & $\begin{array}{l}\text { Alimentação saudável } \\
\text { infantil }\end{array}$ & * & * & * & * & $\begin{array}{l}83,2 \% \\
(\mathrm{n}=505)\end{array}$ & $\begin{array}{l}16,8 \% \\
(n=102)\end{array}$ & $\begin{array}{l}0 \% \\
(\mathrm{n}=0)\end{array}$ & * \\
\hline 2010/2011 & $\begin{array}{l}\text { Alimentação saudável } \\
\text { infantil }\end{array}$ & * & * & * & * & $\begin{array}{l}70,5 \% \\
(\mathrm{n}=217)\end{array}$ & $\begin{array}{l}29,2 \% \\
(\mathrm{n}=90)\end{array}$ & $\begin{array}{l}0,3 \% \\
(n=1)\end{array}$ & ++ \\
\hline $2013 / 2014$ & Lanches saudáveis & $\begin{array}{l}\text { Construção de lanches } \\
\text { saudáveis }\end{array}$ & $\mathrm{O}$ & * & * & * & * & * & * \\
\hline \multirow{5}{*}{$2014 / 2015$} & \multirow{5}{*}{$\begin{array}{l}\text { Pequeno-almoço e } \\
\text { lanches saudáveis }\end{array}$} & Ingestão de PA & $\uparrow$ & \multirow{5}{*}{ * } & \multirow{5}{*}{ * } & \multirow{5}{*}{ * } & \multirow{5}{*}{ * } & \multirow{5}{*}{ * } & \multirow{5}{*}{ * } \\
\hline & & Ingestão de fruta ao PA & $\uparrow \uparrow \uparrow$ & & & & & & \\
\hline & & $\begin{array}{l}\text { Ingestão de refrigerantes e } \\
\text { sumos de fruta ao PA }\end{array}$ & $\downarrow$ & & & & & & \\
\hline & & Ingestão de cereais ao PA & $\mathrm{O}$ & & & & & & \\
\hline & & Realizar lanches saudáveis & $\mathrm{O}$ & & & & & & \\
\hline \multirow{4}{*}{$2015 / 2016$} & \multirow{4}{*}{$\begin{array}{l}\text { Lanche VENCE - } \\
\text { Variado, Económico, } \\
\text { Nutritivo e Equilibrado }\end{array}$} & $\begin{array}{l}\text { Ingestão de hortofrutícolas } \\
\text { ao lanche }\end{array}$ & $\uparrow$ & \multirow{4}{*}{ * } & \multirow{4}{*}{ * } & \multirow{4}{*}{ * } & \multirow{4}{*}{ * } & \multirow{4}{*}{ * } & \multirow{4}{*}{ * } \\
\hline & & $\begin{array}{l}\text { Ingestão de sumos/ } \\
\text { refrigerantes ao lanche }\end{array}$ & $\downarrow$ & & & & & & \\
\hline & & $\begin{array}{l}\text { Ingestão de pão de leite/pão } \\
\text { de forma ao lanche }\end{array}$ & $\downarrow$ & & & & & & \\
\hline & & $\begin{array}{l}\text { Ingestão de bolachas e bolos } \\
\text { ao lanche }\end{array}$ & $\downarrow$ & & & & & & \\
\hline \multirow{4}{*}{$2016 / 2017$} & \multirow{4}{*}{ Dieta Mediterrânica } & Ingestão de PA & $\uparrow$ & \multirow{4}{*}{$\begin{array}{l}\text { Conhecimentos } \\
\text { sobre Dieta } \\
\text { Mediterrânica }\end{array}$} & & & & & \\
\hline & & Ingestão de lanches & $\uparrow$ & & & & & & \\
\hline & & Adesão à Dieta Mediterrânica & $\uparrow$ & & 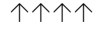 & $(n=182)$ & $(\mathrm{n}=36)$ & $(\mathrm{n}=5)$ & $\mathrm{O}$ \\
\hline & & $\begin{array}{l}\text { Envolvimento no planeamento } \\
\text { e preparação das refeições }\end{array}$ & $\mathrm{O}$ & & & & & & \\
\hline & & Ingestão de PA & $\downarrow$ & & & & & & \\
\hline & & Ingestão de lanches & $\uparrow$ & Conhecimentos & & & & & \\
\hline $2017 / 2018$ & Dieta Mediterrânica & Adesão à Dieta Mediterrânica & $\uparrow$ & sobre Dieta & $\uparrow \uparrow \uparrow$ & $\begin{array}{l}50,5 \% \\
(n=53)\end{array}$ & $\begin{array}{l}39,1 \% \\
(n=41)\end{array}$ & $\begin{array}{l}10,5 \% \\
(n=11)\end{array}$ & +++ \\
\hline & & $\begin{array}{l}\text { Envolvimento no planeamento } \\
\text { e preparação das refeições }\end{array}$ & $\uparrow$ & & & & & & \\
\hline & Dieta Mediterrânica & PA completo e saudável b & $\uparrow$ & Conhecimentos & & & & & \\
\hline 2018/2019 & e Pequeno-almoço & Ingestão de fruta ao PA & 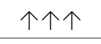 & $\begin{array}{c}\text { sobre Dieta } \\
\text { Mediterrânica e o }\end{array}$ & 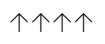 & $\begin{array}{l}39,0 \% \\
(\mathrm{n}=80)\end{array}$ & $\begin{array}{l}58,0 \% \\
(\mathrm{n}=119)\end{array}$ & $\begin{array}{l}2,9 \% \\
(n=6)\end{array}$ & ++ \\
\hline & saudável & Ingestão de cereais de PA & $\mathrm{O}$ & pequeno-almoço & & & & & \\
\hline $\begin{array}{l}\text { O: Sem efeito } \\
\star \text { : Sem dados } \\
\text { a: Excesso de } \\
\text { b: Pelo menos } \\
\text { PA: Pequeno-a }\end{array}$ & $\begin{array}{l}\text { peso incluindo obesidade } \\
2 \text { vezes por semana } \\
\text { almoço }\end{array}$ & $\begin{array}{l}\text { Efeito após } \\
\uparrow: 1 \% \text { a } 5 \% \\
\uparrow \uparrow: 6 \% \text { a } \\
\uparrow \uparrow \uparrow: 119 \\
\uparrow \uparrow \uparrow \uparrow:> \\
\downarrow:-1 \% \text { a }\end{array}$ & $\begin{array}{l}-9 \text { meses: } \\
10 \% \\
\text { a } 15 \% \\
5 \% \\
\%\end{array}$ & & & $\begin{array}{l}\text { Efeito após } 1 \\
+: 5 \% \text { a } 10 \% \\
++: 11 \% \text { a } 1 \\
+++:>15 \%\end{array}$ & meses: & & \\
\hline
\end{tabular}

valores médios de peso superiores, o que se traduziu de igual forma em valores médios de $\mathrm{IMC}\left(\mathrm{kg} / \mathrm{m}^{2}\right)$ superiores nas raparigas.

A prevalência de excesso de peso (incluindo obesidade) mostrou uma tendência decrescente de 2008 a 2019 embora se verifiquem ligeiras variações nos anos letivos 2010/2011 e 2017/2018. Na década em estudo, observou-se um decréscimo de $11,2 \%$ de crianças com excesso de peso (35,5\% para $24,3 \%$ ) e de $10,3 \%$ na prevalência de obesidade infantil (16,7\% para 6,4\%)

Comparativamente aos rapazes, as raparigas apresentam prevalências de excesso de peso superiores em todos os anos letivos.

Analisando o primeiro e último ano observa-se uma redução do excesso de peso em 12,3\% (de 33,4\% para 21,1\%) e 8,3\% (de 37,2\% para $28,9 \%$ ) nos sexos masculino e feminino, respetivamente.

Relativamente à prevalência de obesidade entre 2018 e 2019 verificou-se, igualmente, uma diminuição de 10,8\% nos rapazes (de $17,8 \%$ para $7 \%$ ) e $10,3 \%$ nas raparigas (de $15,9 \%$ para $5,6 \%$ ).

De referir que, foram verificadas diferenças estatisticamente significativas entre os anos letivos 2008/2009 e 2018/2019 do programa MUN-SI, nas classes de estado nutricional referentes ao excesso de peso e obesidade $(p<0,05)$ (Gráfico 1).

\section{DISCUSSÃO DOS RESULTADOS}

O programa MUN-SI é o único programa de promoção para a saúde em Portugal que partilha experiências há mais de uma década com outros países em Redes Europeias como a EPODE (IEN) (19) e a EPHNA (European Public Health Nutrition Alliance) (20) fundamentando-se em estudos de base científica que demonstram que é possível reverter a problemática da malnutrição infantil através de ações ao nível comunitário (19).

O presente estudo demonstrou que o programa MUN-SI contribuiu, de uma forma geral, positivamente para a mudança comportamental das crianças participantes nas Escolas do 1CEB de Oeiras, destacando-se o aumento do consumo de hortofrutícolas principalmente no momento dos lanches, $+2,3 \%$ em 2015/2016, e ao pequeno-almoço: $+16 \%$ em $2014 / 2015$ e $+11,9 \%$ em 2017/2018. Importa referir que o Regime de Fruta Escolar (21), estratégia nacional implementada desde 2009 nos municípios aderentes, tem sido um forte aliado e promotor que tem vindo a potencializar a ação do MUN-SI e de outras iniciativas igualmente bem-sucedidas (17). Relativamente à adesão à Dieta Mediterrânica no MUN-SI, temática enfatizada nos últimos anos de intervenção (2016 a 2019), verificou-se que o efeito positivo observado 
Tabela 3

Valores médios de estatura (cm), peso $(\mathrm{kg})$ e IMC (kg/m²) de crianças dos 6-10 anos no programa MUN-SI, de 2008-2019

\begin{tabular}{|c|c|c|c|c|c|c|c|c|c|c|c|c|c|c|}
\hline & \multirow{2}{*}{ SEXO } & \multicolumn{4}{|c|}{$2008 / 2009$} & \multicolumn{3}{|c|}{$2010 / 2011$} & \multicolumn{3}{|c|}{$2017 / 2018$} & \multicolumn{3}{|c|}{$2018 / 2019$} \\
\hline & & IDADE & $\mathrm{N}$ & MÉDIA & DP & $\mathrm{N}$ & MÉDIA & DP & $\mathrm{N}$ & MÉDIA & DP & $\mathrm{N}$ & MÉDIA & DP \\
\hline \multirow{12}{*}{ Estatura $(\mathrm{cm})$} & \multirow{6}{*}{ Masculino } & 6 & 5 & 120,6 & 2,6 & - & - & - & - & - & - & - & - & - \\
\hline & & 7 & 230 & 127,6 & 5,0 & - & - & - & - & - & - & - & - & - \\
\hline & & 8 & 147 & 130,6 & 5,9 & - & - & - & - & - & - & 47 & 132,4 & 5,9 \\
\hline & & 9 & 9 & 133,8 & 6,1 & 93 & 138,9 & 5,9 & 4 & 138,0 & 12,0 & 49 & 135,5 & 6,1 \\
\hline & & 10 & 6 & 142,5 & 12,5 & 78 & 141,5 & 5,9 & 98 & 137,7 & 6,2 & 32 & 140,0 & 7,1 \\
\hline & & Total & 397 & 129,0 & 6,0 & 171 & 140,1 & 6,0 & 102 & 137,7 & 6,4 & 128 & 135,5 & 6,9 \\
\hline & \multirow{6}{*}{ Feminino } & 6 & 3 & 124,0 & 5,0 & - & - & - & - & - & - & - & - & - \\
\hline & & 7 & 273 & 127,0 & 5,9 & - & - & - & - & - & - & - & - & - \\
\hline & & 8 & 162 & 129,6 & 5,9 & - & - & - & - & - & - & 40 & 131,3 & 5,1 \\
\hline & & 9 & 7 & 132,0 & 10,2 & 131 & 138,6 & 6,7 & 4 & 134,3 & 1,3 & 39 & 135,5 & 5,9 \\
\hline & & 10 & 2 & 135,0 & 12,7 & 115 & 142,1 & 7,5 & 89 & 140,5 & 7,2 & 11 & 139,9 & 6,4 \\
\hline & & Total & 447 & 128,0 & 6,2 & 246 & 140,3 & 7,3 & 93 & 140,2 & 7,1 & 90 & 134,2 & 6,3 \\
\hline \multirow{12}{*}{ Peso (kg) } & \multirow{6}{*}{ Masculino } & 6 & 5 & 23,0 & 2,1 & - & - & - & - & - & - & - & - & - \\
\hline & & 7 & 230 & 28,2 & 5,6 & - & - & - & - & - & - & - & - & - \\
\hline & & 8 & 147 & 29,9 & 6,7 & - & - & - & - & - & - & 47 & 28,6 & 4,8 \\
\hline & & 9 & 9 & 32,7 & 6,3 & 93 & 34,8 & 6,9 & 4 & 38,8 & 15,7 & 49 & 31,6 & 6,4 \\
\hline & & 10 & 6 & 36,8 & 11,1 & 78 & 36,3 & 9,2 & 98 & 33,7 & 6,9 & 32 & 33,4 & 5,0 \\
\hline & & Total & 397 & 29,0 & 6,265 & 171 & 35,5 & 8,0 & 102 & 33,9 & 7,4 & 128 & 30,9 & 5,8 \\
\hline & \multirow{6}{*}{ Feminino } & 6 & 3 & 28,7 & 5,1 & - & - & - & - & - & - & - & - & - \\
\hline & & 7 & 273 & 28,7 & 6,3 & - & - & - & - & - & - & - & - & - \\
\hline & & 8 & 162 & 29,9 & 6,6 & - & - & - & - & - & - & 40 & 29,8 & 5,1 \\
\hline & & 9 & 7 & 39,0 & 20,8 & 131 & 36,6 & 8,5 & 4 & 32,8 & 3,6 & 39 & 31,3 & 6,3 \\
\hline & & 10 & 2 & 31,0 & 9,9 & 115 & 38,9 & 10,5 & 89 & 37,3 & 9,4 & 11 & 36,3 & 6,4 \\
\hline & & Total & 447 & 29,30 & 6,914 & 246 & 37,7 & 9,5 & 93 & 37,1 & 9,3 & 90 & 31,2 & 6,1 \\
\hline \multirow{12}{*}{ IMC $\left(\mathrm{kg} / \mathrm{m}^{2}\right)$} & \multirow{6}{*}{ Masculino } & 6 & 5 & 15,9 & 1,2 & - & - & - & - & - & - & - & - & - \\
\hline & & 7 & 230 & 17,2 & 2,6 & - & - & - & - & - & - & - & - & - \\
\hline & & 8 & 147 & 17,4 & 3,0 & - & - & - & - & - & - & 47 & 16,2 & 2,0 \\
\hline & & 9 & 9 & 18,1 & 3,1 & 93 & 18,0 & 2,9 & 4 & 19,7 & 4,5 & 49 & 17,1 & 2,7 \\
\hline & & 10 & 6 & 17,9 & 3,7 & 78 & 17,9 & 3,3 & 98 & 17,7 & 2,8 & 32 & 17,0 & 1,9 \\
\hline & & Total & 397 & 17,3 & 2,8 & 171 & 18,0 & 3,1 & 102 & 17,8 & 2,9 & 128 & 16,8 & 2,3 \\
\hline & \multirow{6}{*}{ Feminino } & 6 & 3 & 18,7 & 2,0 & - & - & - & - & - & - & - & - & - \\
\hline & & 7 & 273 & 17,7 & 2,9 & - & - & - & - & - & - & - & - & - \\
\hline & & 8 & 162 & 17,7 & 3,0 & - & - & - & - & - & - & 40 & 17,2 & 2,3 \\
\hline & & 9 & 7 & 21,5 & 7,5 & 131 & 18,9 & 3,3 & 4 & 18,2 & 2,1 & 39 & 17,0 & 2,6 \\
\hline & & 10 & 2 & 16,6 & 1,9 & 115 & 19,1 & 3,9 & 89 & 18,7 & 3,5 & 11 & 18,5 & 2,7 \\
\hline & & Total & 447 & 17,7 & 3,0 & 246 & 19,0 & 3,5 & 93 & 18,7 & 3,5 & 90 & 17,3 & 2,5 \\
\hline
\end{tabular}

DP: Desvio-padrão

IMC: Índice de massa corporal

nestes 3 anos escolares, principalmente no ano 2016/2017 (+20,9\%) foi semelhante ao reportado pelo projeto "Eat Mediterranean (EAT)" (22) , uma iniciativa implementada em 2015 e 2016 em 2 municípios portugueses em meio escolar, numa faixa etária mais ampla (3-18 anos), onde igualmente ao fim de 1 ano de intervenção se observou uma adesão à $\mathrm{DM}$ de $+17,6 \%$ das crianças participantes (17). O mesmo programa demonstrou ainda resultados positivos relativamente à redução do consumo de cereais de pequeno-almoço (22) efeito que não se observou no programa MUN-SI em crianças do 1CEB, reforçando a necessidade de trabalhar em estratégias inovadoras que abordem esta temática.

Neste tipo de programas de base comunitária, o impacto positivo nos indicadores medidos deve-se, na sua maioria, pela continuidade e recurso a sessões de educação alimentar em grupo que desencorajam o consumo de itens alimentares menos saudáveis como está patente e de forma transversal a todos os anos do programa MUN-SI. De forma lúdico-educativa, estas sessões parecem contribuir para uma menor preferência de alimentos com alto teor de açúcar como refrigerantes, bolachas e outros produtos de pastelaria substituindo-os por fruta fresca, pela sua disponibilidade e conveniência, relativamente a outros grupos de alimentos, como os hortícolas ou as leguminosas que parecem ser menos atrativos nomeadamente para o momento dos lanches e como foi igualmente confirmado no estudo "Children Study" (23).

As intervenções ao nível do aumento da Atividade Física parecem ser uma estratégia bastante recorrente nos programas municipais já que é fácil de implementar e de mobilizar a comunidade, são necessárias poucas ferramentas e recursos, e é bastante acessível e disponível em vários espaços (24). No MUN-SI, foi verificado o efeito positivo da intervenção no aumento da prática de atividade física nas crianças (+11 a 15\% na prática de AF regular) que foi igualmente encontrado em programas com as mesmas metodologias nomeadamente $\mathrm{O}$ "Children Study" (23) na Grécia e no EAT em Portugal (25). Com a orientação de programas como o MUN-SI, a escola oferece, assim, as infraestruturas e os meios para que o aumento da AF em crianças seja bem-sucedido e efetivo. 
Prevalência de excesso de peso e obesidade das crianças no programa MUN-SI, por género, nos anos letivos 2008/2009, 2010/2011, $2017 / 2018$ e $2018 / 2019$

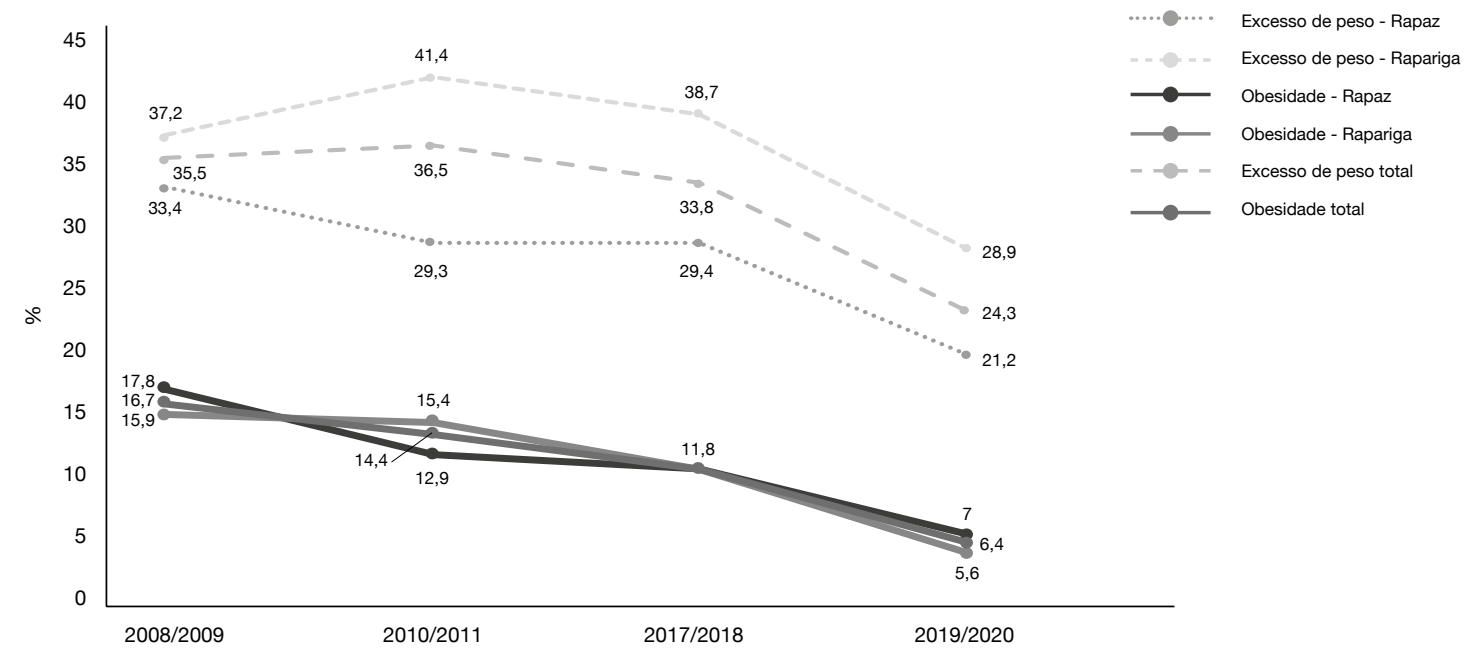

Grupo etários considerados: 2008/2009 (7-8 anos), 2010/2011 (9 e 10 anos), 2017/2018 (9 e 10 anos) e 2018/2019 (8-10 anos).

* Diferença entre os anos letivos 2008/2009 e 2018/2019 é estatisticamente significativa $(p<0,05)$

O sucesso de intervenções que combinam vários parâmetros foi descrito numa revisão que reúne 71 programas europeus de base comunitária (11). Nestes observou-se que cerca de $70 \%$ avaliaram o impacto das intervenções no estado nutricional em crianças dos 6 aos 12 anos, tendo-se confirmado um efeito positivo na maioria dos programas, muito embora se reconheçam as limitações associadas ao desenho das intervenções entre estas a ausência de grupo controlo, a falta de aleatorização e baixo tamanho amostral, exatamente os mesmos grupos etários nos anos dos programas, limitações estas que estão igualmente subjacentes na metodologia do programa MUN-SI. Em Portugal, a tendência decrescente da prevalência de excesso de peso e obesidade em crianças dos 6 aos 10 anos tem vindo a ser reportada pelo estudo COSI Portugal $(4,11)$. Esta tendência foi ainda mais evidente no programa MUN-SI, que seguiu a mesma metodologia de avaliação, e foi implementado em igual período e nas mesmas faixas etárias.

Como seria de esperar, os valores médios de estatura, peso e IMC aumentaram com a idade e ao longo da década (2008 a 2019). O decréscimo no excesso de peso (EP) e obesidade (OB) infantil foi mais acentuado no MUN-SI do que no COSI Portugal (4), o programa de vigilância nutricional infantil do Ministério da Saúde, realizado a cada 3 anos em crianças dos 6 aos 8 anos, igualmente desde 2008.

$\mathrm{Na}$ década em estudo, o MUN-SI confirmou um decréscimo de $11,2 \%$ de crianças com excesso de peso (de $35,5 \%$ para $24,3 \%$ ) comparativamente aos $8,3 \%$ de EP (37,9\% para 29,6\%), observados no COSI (4). Esta diferença foi ainda mais acentuada na prevalência de obesidade já que de 2008 para 2019 houve um decréscimo de $10,3 \%$ de crianças com obesidade infantil $(16,7 \%$ para $6,4 \%)$ no MUN-SI comparativamente com o decréscimo de 3,3\% (15,3\% para $12 \%)$ nas crianças do COSI (4).

Em relação aos dados apresentados por sexo, comparativamente aos rapazes, as raparigas participantes no MUN-SI apresentaram prevalências de excesso de peso (EP) e de obesidade (OB), superiores em todos os anos letivos, tendo-se observado uma redução de 8,3\% de EP (de $37,2 \%$ para $28,9 \%$ ) nas raparigas e $12,3 \%$ de EP (de $33,4 \%$ para $21,1 \%)$ nos rapazes. Entre os mesmos anos, embora o estudo COSI Portugal tenha mostrado a mesma tendência invertida, a prevalência de excesso de peso foi, no entanto, maior nos rapazes dos que nas raparigas muito embora o decréscimo tenha sido mais acentuado no sexo masculino à semelhança do MUN-SI. Assim, de 2008 para 2019, o estudo COSI Portugal mostrou um decréscimo na prevalência de EP nas raparigas de $7 \%$ (36,5\% para 29,5\%) e de $9 \%$ (39,3\% para $29,6 \%)$ nos rapazes (4).

Relativamente à prevalência de obesidade, verificou-se um decréscimo semelhante por sexo, embora mais acentuado no MUN-SI (-10,8\% rapazes e - 10,3\% raparigas) do que no COSI Portugal (-3,4\% rapazes e $-3,1 \%$ raparigas) (4).

Ressalva-se que a amostra de escolas que participaram no programa MUN-SI, não é representativa a nível municipal, regional ou nacional pelo que estes dados deverão ser analisados com algum cuidado, valorizando, no entanto aquele que parece ser o efeito mais acentuado do MUN-SI, enquanto programa de intervenção, na abordagem do excesso de peso infantil, quando comparado com um estudo de vigilância como o COSI Portugal. Neste contexto concorre para o sucesso do MUN-SI o seu caráter multidimensional, multidisciplinar e de continuidade do programa na mesma comunidade, não somente durante toda a programação de atividades contínuas dentro do ano letivo mas ao longo do tempo, principalmente ao longo de uma década, considerando-se um exemplo singular e pouco conhecido em Portugal. Reforça-se ainda que o MUN-SI foi desenhado e desenvolvido com base em iniciativas bem-sucedidas e sustentáveis, verificados em programas comunitários da Europa, como o exemplo Francês (estudo FLVS) (26) no qual foram realizadas múltiplas intervenções na abordagem da obesidade infantil, com sucesso, intervenções estas de base escolar no âmbito da nutrição de forma contínua ao longo de 12 anos.

\section{CONCLUSÕES}

Os resultados que o programa MUN-SI revelam após uma década, sugerem que programas de intervenção de base comunitária de longa duração e que abrangem vários agentes envolvidos no meio escolar (auxiliares educativos, profissionais de educação, família, crianças, município), poderão ter um efeito sinérgico no que diz respeito à diminuição da prevalência de excesso de peso e obesidade infantil, e mais diretamente no aumento de conhecimentos sobre a alimentação saudável, concretamente sobre os benefícios e adesão à Dieta 
Mediterrânica, tendo-se verificado ainda mudança comportamental ao nível da atividade física e de aumento no consumo de hortofrutícolas, em crianças. Estes dados reforçam o potencial destas iniciativas e justificam a prioridade atribuível à formulação e ao desenvolvimento de programas de ação com abrangência nacional, que estimulem a adoção de estilos de vida mais saudáveis e criem condições estruturais e ambientais favoráveis à saúde infantil.

Neste contexto, é necessário recorrer a estratégias diversificadas e complementares de intervenção, de que são exemplo o "empoderamento" dos cidadãos, o desenvolvimento de competências pessoais, a criação de ambientes favoráveis à saúde e o delineamento de políticas saudáveis.

A resolução deste problema, considerada a multiplicidade dos respetivos determinantes, a globalização da sua expressão e a diversidade das intervenções nas respostas requer o estabelecimento de parcerias funcionais e de alianças e redes fortes para a promoção da saúde.

\section{AGRADECIMENTOS}

Os autores gostariam de agradecer a todos os nutricionistas colaboradores, e aos que implementaram o MUN-SI de forma mais direta até 2018, em particular à Ana Lúcia Silva, Emília Alves, Rita Garcia e Inês Portugal, aos técnicos municipais, crianças, pais, encarregados de educação, professores e auxiliares de educação pela sua contribuição na organização e implementação do Programa MUN-Si assim como ao Ministério da Educação (Direção-Geral de Educação) e Ministério da Saúde (Direção-Geral da Saúde e Instituto Nacional de Saúde Doutor Ricardo Jorge, IP) pelo seu inestimável apoio institucional.

\section{REFERÊNCIAS BIBLIOGRÁFICAS}

1. Abarca-Gómez L, Abdeen ZA, Hamid ZA, Abu-Rmeileh NM, Acosta-Cazares B, Acuin C, et al. Worldwide trends in body-mass index, underweight, overweight, and obesity from 1975 to 2016 : a pooled analysis of 2416 population-based measurement studies in 128•9 million children, adolescents, and adults. 2017;6736(17):321293. Available from: https://www.thelancet.com/journals/lancet/article/PIIS01406736(17)32129-3/fulltext.

2. World Health Organization/Regional Office for Europe. Childhood Obesity Surveillance Initiative (COSI) Protocol [Internet]. 2016. Available from: https://www.euro.who.int/_ data/assets/pdf_file/0018/333900/COSI-protocol-en.pdf.

3. Rito A, Wijnhoven TMA, Rutter H, Carvalho MA, Paixão E, Ramos C, et al. Prevalence of obesity among Portuguese children (6-8 years old) using three definition criteria: COSI Portugal, 2008. Pediatric Obesity. 2012;7(6):413-22.

4. Childhood Obesity Surveillance Initiative - COSI Portugal 2019 Factsheet [Internet]. 2019. Available from: http://www.ceidss.com/wp-content/uploads/2019/07/COSI2019_-FactSheet.pdf.

5. World Health Organization/Regional Office for Europe. Childhood Obesity Surveillance Initiative HIGHLIGHTS 2015-17 [Internet]. Copenhagen; 2018. Available from: http://www.euro.who.int/_data/assets/pdf_file/0006/372426/WH14_COSI_ factsheets_v2.pdf?ua=1.

6. Rito Al, Buoncristiano M, Spinelli A, Salanave B, Kunešová M, Hejgaard T, et al. Association between Characteristics at Birth, Breastfeeding and Obesity in 22 Countries : The WHO European Childhood Obesity Surveillance Initiative - COSI 2015 / 2017. 2019;226-43. Available from: https://www.karger.com/Article/FullText/500425. 7. Spinelli A, Buoncristiano M, Kovacs VA, Yngve A, Spiroski I, Obreja G, et al. Prevalence of Severe Obesity among Primary School Children in 21 European Countries. 2019;24458. Available from: https://www.karger.com/Article/FullText/500436.

8. Rito Al, Carvalho MA, Ramos C. Program Obesity Zero (POZ) - a communitybased intervention to address overweight primary-school children from five Portuguese municipalities Public Health Nutrition. 2013;(8):1-9.
9. Gortmaker SL, Swinburn BA, Levy D, Carter R, Mabry PL, Finegood DT, et al. Changing the future of obesity : Science, policy, and action. The Lancet [Internet]. 2011;378(9793):838-47. Available from: http://dx.doi.org/10.1016/S01406736(11)60815-5.

10. World Health Organization. Report of the Commission on Ending Childhood Obesity [Internet]. Geneva; 2016. Available from: https://apps.who.int/iris/bitstream/ handle/10665/204176/9789241510066_eng.pdf?sequence=1.

11. Bemelmans W, Wijnhoven T, Verschuuren M, Breda J. Overview of 71 European community-based initiatives against childhood obesity starting between 2005 and 2011 : general characteristics and reported effects. BMC Public Health. 2014;14(1):758. 12. Borys J, le Bodo Y, de Henauw S, Moreno L, Romon M, Seidell J. Preventing Childhood obesity: EPODE European Network Recommendations. Lavoisier. 2011. 265. 13. MUN-SI. MUN-SI [Internet]. [cited 2020 Oct 28]. Available from: http://mun-si.com/. 14. Carvalho M, Ramos C, Breda J, Rito A. "Zero Obesity Questionnaire" (ZOQ) - The development and validation of a novel questionnaire to assess nutrition knowledge, food behaviours and self image in school children. In: "The 4th Scandinavian Pediatric Obesity Conference Poster Presentations [Internet]. 2010. p. 416. Available from: http:// onlinelibrary.wiley.com/doi/10.1111/j.1467-789X.2010.00763_7.x/pdf.

15. World Health Organization/Regional Office for Europe. Childhood Obesity Surveillance Initiative (COSI) - Data collection procedures. 2016.

16. World Health Organization. WHO Child Growth standards: Training Course on Child Growth Assessment. [Internet]. Geneva; 2008. Available from: http://www.who. int/childgrowth/training/en.

17. Rito Al, Dinis A, Rascôa A, Maia A, Mender S, Stein-Novais C, et al. Mediterranean Diet Index ( KIDMED ) Adherence, Socioeconomic Determinants, and Nutritional Status of Portuguese Children : The Eat Mediterranean Program. Portuguese Journal of Public Health [Internet]. 2019;36. Available from: https://www.karger.com/Article/ FullText/495803.

18. World Health Organization. Physical activity and young people [Internet]. [cited 2020 Feb 28]. Available from: https://www.who.int/dietphysicalactivity/factsheet_ young_people/en/.

19. Borys J, Bodo Y le, Jebb SA, Seidell JC, Summerbell C, Richard D, et al. EPODE approach for childhood obesity prevention : methods, progress and international development. 2012;(April):299-315.

20. European Public Health Nutrition Alliance. European Public Health Nutrition Alliance [Internet]. [cited 2020 Feb 28]. Available from: http://www.ephna.eu/.

21. Direção-Geral dos Estabelecimentos Escolares (Dgeste). Regime de Fruta Escolar [Internet]. [cited 2020 Feb 28]. Available from: https://www.dgeste.mec.pt/index.php/ regime-de-fruta-escolar.

22. Rito Al, Dinis A, Rascôa C, Maia A, Carvalho I de, Mariana M, et al. Improving breakfast patterns of portuguese children - an evaluation of ready-to-eat cereals according to the European nutrient pro fi le model. European Journal of Clinical Nutrition. 2018;(77).

23. Angelopoulos PD, Milionis HJ, Grammatikaki E, Moschonis G, Manios Y. Changes in BMl and blood pressure after a school based intervention : The CHILDREN study. European Journal of Public Health. 2009;319-25.

24. Rito Al, Cardoso R, Portugal I, Baleia J, Mendes S, Bica M. Evaluation of Portuguese Community Health Projects and initiatives within the European and National Healthy Cities Network. Acta Portuguesa de Nutrição [Internet]. 2019;(16):4-8. Available from: http://actaportuguesadenutricao.pt/wp-content/uploads/2019/05/02_Evaluation-ofPortuguese-community-health-projects-and-initiatives-within-the-European-andNational-Healthy-Cities-Network.pdf.

25. Lima A, Baleia J, Mendes S, Portugal I, Rascôa C, Dinis A, et al. Prática de atividade física em crianças e adolescentes do universo escolar de dois municípios portugueses - Projeto "EAT MEDITERRANEAN." In: Acta Portuguesa de Nutrição [Internet]. 2019. p. 89. Available from: https://actaportuguesadenutricao.pt/wp-content/uploads/2019/07/ resumos_PO_XVIIICNA.pdf.

26. Romon M, Lommez A, Tafflet M, Basdevant A, Charles MA, Oppert JM, et al. Downward trends in the prevalence of childhood overweight in the setting of 12-year school- and community-based programmes. 2008;12(10):1735-42. 